\title{
DISCRIMINATIVE SLIDING PRESERVING REGULARIZATION IN MEDICAL IMAGE REGISTRATION
}

\author{
Dan Ruan $^{1,2}$, Selim Esedō̄lu ${ }^{1}$, Jeffrey A. Fessler ${ }^{1}$ \\ ${ }^{1}$ The University of Michigan, Ann Arbor, MI \\ ${ }^{2}$ Stanford University, Stanford, CA
}

\begin{abstract}
Sliding effects often occur along tissue/organ boundaries. For instance, it is widely observed that the lung and diaphragm slide against the rib cage and the atria during breathing. Conventional homogeneous smooth registration methods fail to address this issue. Some recent studies preserve motion discontinuities by either using joint registration/segmentation or utilizing robust regularization energy on the motion field. However, allowing all types of discontinuities is not strict enough for physical deformations. In particular, flows that generate local vacuums or mass collisions should be discouraged by the energy functional. In this study, we propose a regularization energy that encodes a discriminative treatment of different types of motion discontinuities. The key idea is motivated by the Helmholtz-Hodge decomposition, and regards the underlying motion flow as a superposition of a solenoidal component, an irrotational component and a harmonic part. The proposed method applies a homogeneous penalty on the divergence, discouraging local volume change caused by the irrotational component, thus avoiding local vacuum or collision; it regularizes the curl field with a robust functional so that the resulting solenoidal component vanishes almost everywhere except on a singular set where the large shear values are preserved. This singularity set corresponds to sliding interfaces. Preliminary tests with both simulated and clinical data showed promising results.
\end{abstract}

Index Terms - registration, divergence, curl

\section{INTRODUCTION}

The ultimate goal of medical image registration is to recover the physical transformation that generated the two (or series) of observation images. The problem of estimating such vector field from noisy intensity observations is ill-posed and necessitates the incorporation of prior knowledge. One effective approach in solving such inverse problems is to use an optimization formulation, where the objective function (total energy) to be minimized is composed of a data (in)fidelity and a regularization term $\left(E=E_{d}+E_{r}\right)$. A well designed regularization energy encodes physical understanding of the desirable properties of the unknown parameters and drives the optimization to solutions with such characteristics. To estimate motion-induced deformation, roughness regularization is commonly used. It penalizes the $L_{2}$ norm of the $k$ th-order derivative of the deformation field ${ }^{1}$. However, when there is sliding along tissue/organ boundaries, homogeneous smoothness regularization leads to estimated transformations that are blurry across the sliding surface - an undesirable artifact.

Recently, several studies $[3,4]$ of joint segmentation and registration have arisen from various disciplines and applications. In these methods, smooth regions and singularity sets (edges) are devised according to image intensity, and registration aims to align each part respectively. As an alternative, [5] proposes to capture the intrinsic discontinuity boundaries incurred by the flow itself, based on a regularization design that interprets he energy minimization process as anisotropic diffusion. Unfortunately, none of the aforementioned methods distinguishes among various discontinuities, and may preserve flow singularities corresponding to dramatic volume change, contradicting our prior knowledge that physical transformations in medical imaging are invertible and roughly volume preserving.

Motivated by the above observations, we study a regularization scheme that preserves large local shear discontinuities and penalizes other types of nonsmoothness. We propose a discriminative regularization energy that encodes the distinction among different contributing components to the Jacobian of the deformation field and use a robust energy functional to implicitly preserve the large shear component. The proposed energy reflects more accurately the physical prior knowledge, and leads to promising results in a preliminary study.

\section{PROPOSED METHOD}

Since the endpoint of regularization design is to penalize solutions that violate physical prior, we start by characterizing the admissible solution set. First, the deformation should be fairly smooth except at the sliding sites. Second, dramatic local volume change seldom occurs in physical deformations

\footnotetext{
${ }^{1}$ Typically $k=1,2$ or their combination is used. If $k=1$, it corresponds to the Horn and Schunck regularization [1], and $k=2$ is related to thin-plate spline formulation [2]. The combination version has an interpretation with the corresponding Sobolev space.
} 
induced by motion and should be excluded; in particular, the deformation should neither map distinct voxels in the source image to the same location in the target image (mass collision) nor the other way around (vacuum). Third, shear (sliding) may be large on a sliding surface (boundary), but should be small within the complement of the motion boundary. Finally, medical image registration involves tissues that are elastic with sliding motion and mild changes in topology, so a physical deformation generally has nonvanishing divergence and curl components everywhere, and we are not interested in the extreme cases of pure solenoidal or irrotational flows. We choose to stay within the optimization framework and seek a proper regularization to drive the solution to a vector field possessing the traits mentioned above, rather than to use serially structured post-processing.

To simplify description and to better motivate the basic ideas, we start by considering the 2-dimensional cases. We represent the components of the deformation field $\Phi$ as $(U, V)$ so that at spatial location $\underline{x}, \Phi(\underline{x})=[U(\underline{x}), V(\underline{x})]$, and the pointwise Jacobian of the deformation is given by

$$
D \Phi(\underline{x})=\left[\begin{array}{ll}
U_{x}(\underline{x}) & U_{y}(\underline{x}) \\
V_{x}(\underline{x}) & V_{y}(\underline{x})
\end{array}\right] .
$$

Recall that the conventional Horn and Schunck type regularization uses the following energy functional:

$$
\begin{aligned}
E_{r, h s} & =\int_{\Omega}\left\{U_{x}^{2}+U_{y}^{2}+V_{x}^{2}+V_{y}^{2}\right\} d \underline{x} \\
& =\int_{\Omega}\|D \Phi\|_{F}^{2} d \underline{x} \\
& =\|\| D \Phi\left\|_{F}\right\|_{2}^{2} .
\end{aligned}
$$

Where the subindex of $\|\cdot\|$ indicates the type of norm applied: $\|\cdot\|_{F}$ corresponds to the Frobenius norm and $\|\cdot\|_{2}$ represents the $L_{2}$ norm. In the above expressions, the components of $D \Phi(\underline{x})$ are combined according to the Frobenius norm; and then the regularization energy is defined as the $L_{2}$ norm (with respect to spatial locations) of this intermediate scalar field.

Note that the trace of the deformation Jacobian, $\operatorname{trace}\{D \Phi\}$, is the divergence of the deformation field, $\nabla \cdot \Phi$,

$$
\operatorname{trace}\{D \Phi\}=U_{x}+V_{y}=\nabla \cdot \Phi,
$$

which characterizes the local volume change. To the first order, $\nabla \cdot \Phi$ agrees with the alternative volume change measure based on local Jacobian $\left(\|D \Phi+I\|_{F}^{2}-1\right)$ used in [6]. This motivates us to derive other quantities from the Jacobian that reflect the physical properties we hope to incorporate.

The Helmholtz-Hodge decomposition [7] states that a smooth vector field can be uniquely decomposed into:

$$
\Phi=\nabla \times f+\nabla g+h,
$$

where $f$ is a vector field and $\nabla \cdot(\nabla \times f)=0 ; g$ is a scalar potential field and that $\nabla \times(\nabla g)=0$; and $h$ is a harmonic

vector field with $\nabla \cdot h=0$ and $\nabla \times h=0$. This implies that the solenoidal component $\nabla \times f$ is the sole contributor to the curl value of the deformation $\nabla \times(\nabla \times f)=\nabla \times \Phi$; and the irrotational component $\nabla g$ is the sole contributor to the divergence value $\nabla \cdot \Phi$. This idea has been explored recently to filter and decompose vector fields [8].

Motived by the above observations, we consider regularizing the divergence and the curl of the deformation field separately, because we could suppress the irrotational component by penalizing $\nabla \cdot \Phi$ and control the behavior of the solenoidal component by regularizing $\nabla \times \Phi$. Note that preservation of mass is a reasonable assumption for physical deformations throughout the region of interest, thus a homogeneous regularization on the divergence component is appropriate. On the other hand, we want to preserve large shear values and suppress the smaller ones caused by observation noise, so it is reasonable to use a robust regularization energy functional on the curl field.

We consider a regularization energy of the form:

$$
\begin{aligned}
E_{r, *, 2 d}(\Phi) & =\int_{\Omega}\left\{\alpha|\nabla \cdot \Phi|^{2}+\beta|\nabla \times \Phi|\right\} d \underline{x} \\
& =\alpha\|\nabla \cdot \Phi\|_{2}^{2}+\beta\|\nabla \times \Phi\|_{1}
\end{aligned}
$$

This is an legitimate expression in 2D since $\nabla \times \Phi$ is a scalar field. Notice that the $L_{1}$ norm on the curl is reminiscent of the total variation formulation in edge preserving image reconstruction. Only that in this case, we are preserving large shears.

In $3 \mathrm{D}$, the deformation $\Phi=[U, V, W]$ is a length-3 vector field, and its divergence and curl components are as follows:

$$
\begin{gathered}
\nabla \cdot \Phi=U_{x}+V_{y}+W_{z} ; \\
\nabla \times \Phi=\operatorname{det}\left[\begin{array}{ccc}
\boldsymbol{i} & \boldsymbol{j} & \boldsymbol{k} \\
\frac{\partial}{\partial x} & \frac{\partial}{\partial y} & \frac{\partial}{\partial z} \\
U & V & W
\end{array}\right],
\end{gathered}
$$

where $\boldsymbol{i}, \boldsymbol{j}, \boldsymbol{k}$ are the unit vectors for the $x-, y-$, and $z$-axes, respectively.

Divergence (3) is still a coordinate independent scalar field as in the 2D case, but the curl component (4) is now a vector field with three coordinates $(\boldsymbol{i}, \boldsymbol{j}, \boldsymbol{k})$ and it is necessary to define an intermediate map to convert this vector field to scalar values in order to apply the suggested regularization functional. We use the $L_{2}$ length as the measure of pointwise strength of the curl field before the spatial integration, ensuring rotatioal invariance of the regularization energy.

$$
\begin{aligned}
E_{r, *, 3 d}(\Phi)= & \alpha\|\nabla \cdot \Phi\|_{2}^{2}+\beta\|\| \nabla \times \Phi\left\|_{2}\right\|_{1} \\
= & \alpha \int_{\Omega}\left(U_{x}+V_{y}+W_{z}\right)^{2} d \underline{x} \\
& +\beta \int_{\Omega} \sqrt{\left(W_{y}-V_{z}\right)^{2}+\left(U_{z}-W_{x}\right)^{2}+\left(V_{x}-U_{y}\right)^{2}} d \underline{x} .
\end{aligned}
$$




\section{PRELIMINARY TEST RESULTS}

For preliminary study, we used voxel based free-form descriptor for the deformation, and the optimization procedure was performed with energy descending variational flows. We have performed the following three sets of tests.

1. As a proof of principle, we started with an initial vector field and updated it such that the proposed regularization energy was decreased. This test was intended as an qualitative justification of the impact of the proposed regularization energy.

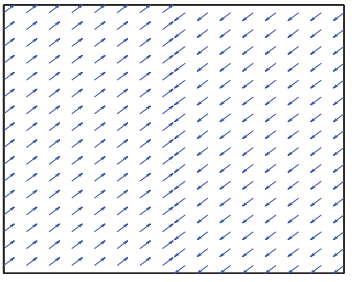

initial flow

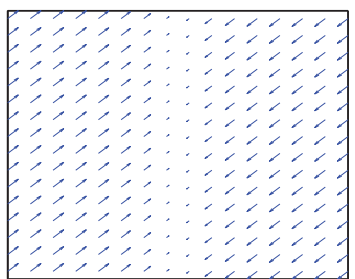

HR regularized flow

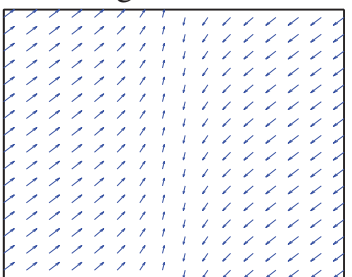

discriminatingly regularized flow

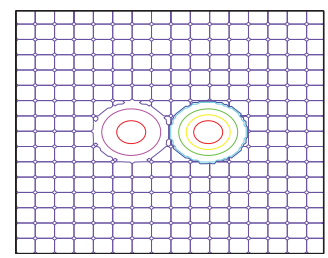

original image

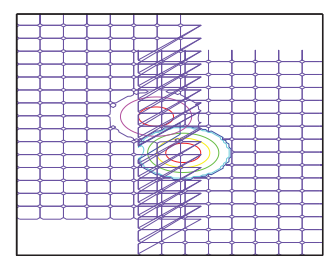

deformed intensity

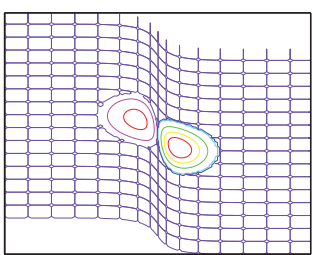

deformed intensity

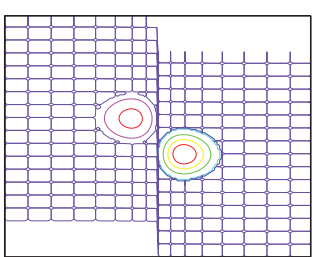

deformed intensity

Fig. 1. Evolution of vector field under descending flow for the regularization energy.

2. For illustrative purposes, we simulated a pair of source $\left(I_{s}\right)$ and target $\left(I_{t}\right)$ images, consisting of two blocks sliding against each other in the vertical direction by 5 pixels. We registered the simulated image pair by minimizing the energy corresponding to both the proposed method and the conventional Horn and Schunck formulation. The sum of squared distance (SSD) was used as

the data discrepancy energy $E_{d}$ so that the overall objective functional to be minimized was given by:

$$
E(\Phi)=E_{d}\left(I_{s}, I_{t}, \Phi\right)+\lambda E_{r}(\Phi)
$$

where the discrepancy energy read

$$
\begin{aligned}
E_{d}\left(I_{s}, I_{t}, \Phi\right) & =\left\|I_{s} \circ(I+\Phi)-I_{t}\right\|_{2}^{2} \\
& =\int_{\Omega}\left(I_{s}(\underline{x}+\Phi(\underline{x}))-I_{t}(\underline{x})\right)^{2} d \underline{x} .
\end{aligned}
$$

Since the local minima depend on the choice of tradeoff parameters $(\lambda, \alpha, \beta)$, we tested a wide variety of parameter values, and compared the best performance among all choices. To illustrate the result for comparison better, we show the value of the vertical component $(V)$ of the estimated deformation field with each regularization method (the truth views as two side-by-side blocks with values -5 and +5 respectively).

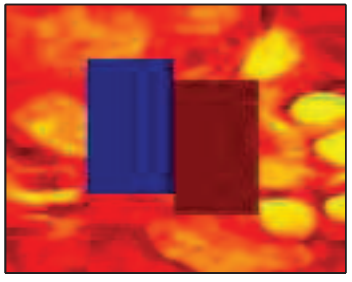

source image

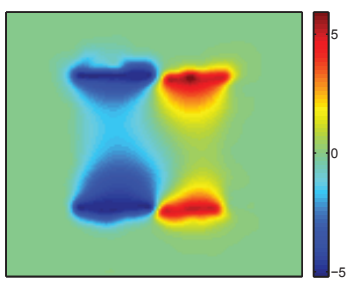

V-comp. with HS reg.

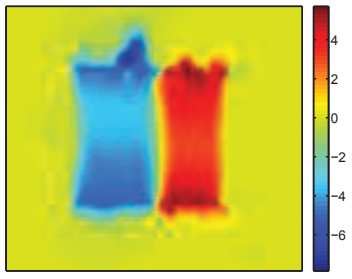

V-comp. w. proposed reg.

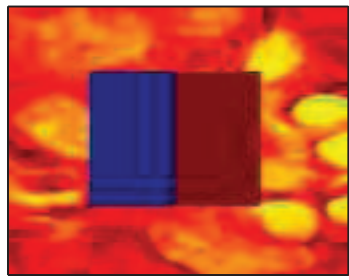

target image

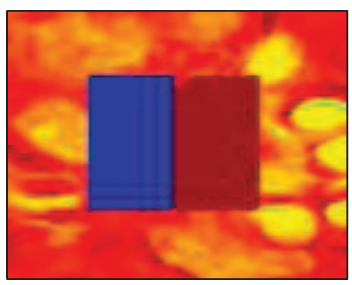

deformed source

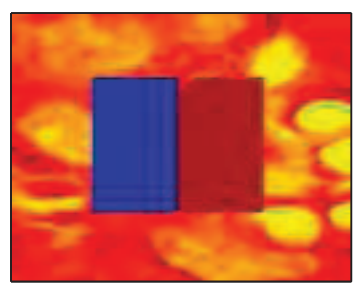

deformed source
Fig. 2. Registration test for simulated sliding blocks.

3. Finally, we performed a comparison test on a thorax CT data set: the images were acquired at deep inhale and deep exhale respectively. The experiment set up were the same as in the simulation test above. In the absence of ground truth, optimal parameter values could not be determined. For a fair comparison, we used a set of tradeoff parameters so that the resulting data discrepancy energy were comparable for both methods, 


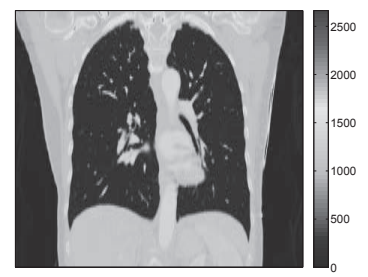

source image

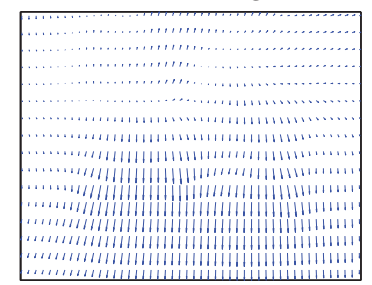

HS registration result

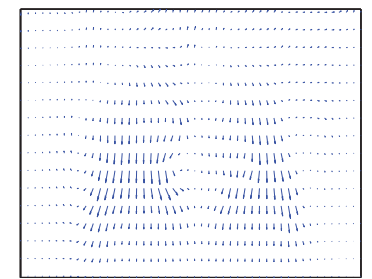

proposed registration result

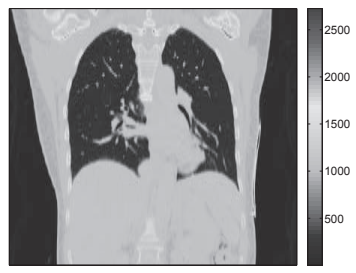

target image

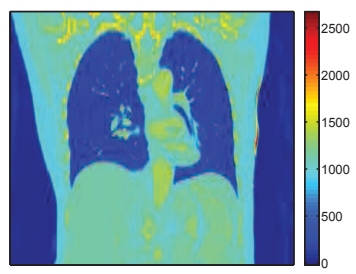

deformed source

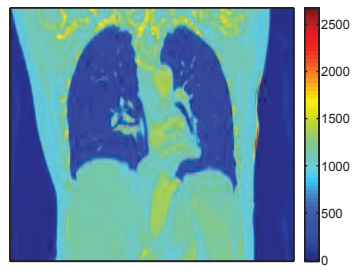

deformed source
Fig. 3. Registration test for thorax CT pair under respiratory motion.

Notice that in both registration cases (test 2 and test 3 ), despite the similarity between deformed intensity images, the proposed regularization led to flow fields that were more physical than the ones estimated with the Horn and Schunck regularization. This further justifies the effect of a well-designed regularization functional: it guides the solution to a local minimum that conforms to prior knowledge.

\section{CONCLUDING REMARKS}

We have proposed a regularization energy that implicitly encodes a discriminative treatment among different types of discontinuities and selectively preserves shear discontinuity boundaries. By penalizing the $L_{2}$ norm of the flow divergence, the proposed method prevents unphysical discontinuities such as mass collisions or vacuums. On the other hand, the $L_{1}$ norm on the curl field drives the deformation to be irrotational for most region of interest, yet allows for shear boundaries. The proposed regularization appropriately reflects our understanding of the sliding effects in medical image registration problems. Preliminary results on both simulated and clinical data were promising. Current work has used the $L_{1}$ normed curl regularization for large shear preserving. Other robust functionals such as Geman-type energy [9], Huber and hyper-geometric functionals are also reasonable choices. We will explore the behavior of those alternative in future work.

Because the objective energy is nonconvex for image registration problems, local minimal solutions are attained. When observations are subject to noise corruption, it is crucial to balance various terms in data fidelity and regularization energy properly - analogous to setting the Tikhonov regularization weight to be inversely proportional to observation noise variance in conventional image denoising problems only that it is much harder in registration problems, due to the nonlinear relationship between the deformation parameter and observed intensity. We are working on a bias-variance analysis, which has the potential to provide guidance for parameter selection.

In the proposed method, the computation complexity for each iteration in the variational PDE evolution is comparable to any alternative regularization methods involving the Jacobian. Moreover, the data fidelity component gives rise to the hyperbolic term in the PDE, which subsequently determines the time step size restricted by the Courant-Friedriches-Lewy (CFL) stability condition. This indicates that the proposed method does not incur intrinsic sacrifice of computation efficiency, compared to alternative regularization methods.

\section{ACKNOWLEDGMENT}

This work is supported in part by the Barbour scholarship from University of Michigan, US NIH grant P01-CA59827, and US NSF DMS-0713767. Dan Ruan acknowledges the support of Radiation Oncology Department at Stanford University. Selim Esedoğlu acknowledges the support of a Sloan Foundation Fellowship.

\section{REFERENCES}

[1] B. Horn and B. Schunck, "Determining optical flow," Artif. Intel., vol. 17, pp. 185-203, 1981.

[2] S. J. Lee, I. T. Hsiao, and G. R. Gindi, "The thin plate as a regularizer in Bayesian SPECT reconstruction," IEEE Trans. Nuc. Sci., vol. 44, no. 3, pp. 1381-7, June 1997.

[3] A. Yezzi, L. Zollei, and T. Kapur, "A variational framework for intergrating segmentation and registration through active contours," Medical Imag. Anal., vol. 7, no. 2, pp. 171-185, Jun. 2003.

[4] M. Droske and M. Rumpf, "Multiscale joint segmentation and registration of image morphology,” IEEE Trans. Patt. Anal. Mech. Intell., vol. 29, no. 12, pp. 2181-2194, Dec. 2007.

[5] D. Ruan, J. A. Fessler, and S. Esedoglu, "Discontinuity preserving regularization for modeling sliding in medical image registration," in Proc. IEEE Nuc. Sci. Symp. Med. Im. Conf., 2008, pp. 5304-5308.

[6] T. Rohlfing, C. R. Maurer, D. A. Bluemke, and M. A. Jacobs, "Volume-preserving nonrigid registration of MR breast images using free-form deformation with an incompressibility constraint," IEEE Trans. Med. Imag., vol. 22, no. 6, pp. 730-741, June 2003.

[7] R. Abraham, J. Marsden, and T. Ratiu, Eds., Manifolds, tensor analysis, and applications, vol. 75 of Applied Mathematical Science, Springer, 1988.

[8] J. Yuan, C. Schnörr, and G. Steidl, "Convex hodge decomposition and regularization of image flows," Preprint, University of Heidelberg, 2008.

[9] S. Geman and D. Geman, "Stochastic relaxation, Gibbs distribution and Bayesian restoration of images," IEEE Trans. Patt. Anal. Mach. Int., vol. 6, no. 6, pp. 721-41, Nov. 1984. 